\title{
INFLUÊNCIA DA EDUCAÇÃO EM SAÚDE NA AUTOEFICÁCIA EM AMAMENTAR: ESTUDO QUASE EXPERIMENTAL
}

\section{THE INFLUENCE OF HEALTH EDUCATION ON BREASTFEED SELF-EFFICACY: QUASI-EXPERIMENTAL STUDY}

\section{LA INFLUENCIA DE LA EDUCACIÓN \\ EN LA SALUD SOBRE LA AUTOEFICACIA EN AMAMANTAR: ESTUDIO CUASI-EXPERIMENTAL}

\author{
Sandra Maria Schultz ${ }^{1}$ \\ Kátia Fernanda Alves Moreira ${ }^{2}$ \\ Priscilla Perez da Silva Pereira ${ }^{3}$ \\ Lerissa Nauana Ferreira ${ }^{4}$ \\ Marcos Antônio Sales Rodrigues 5 \\ Daiana Evangelista Rodrigues Fernandes
}

Como citar este artigo: Schultz SM, Moreira KFA, Pereira PPS, Ferreira LN, Rodrigues MAS, Fernandes DER. Influência da educação em saúde na autoeficácia em amamentar: estudo quase experimental. Rev baiana enferm. 2020;34:35995.

Objetivo: avaliar a intervenção educativa de enfermagem para a promoção da autoeficácia em amamentação em nutrizes internadas em uma maternidade do Norte do Brasil. Método: estudo quase experimental, longitudinal, composto por um grupo de intervenção $(n=80)$ e observacional $(n=78)$. A intervenção educativa deu-se mediante roda de conversa. A coleta de dados ocorreu no período de janeiro a março de 2018. Resultados: no grupo de intervenção as nutrizes apresentaram alta eficácia para a amamentação, enquanto que no grupo de observação encontrou-se moderada eficácia. Entre as nutrizes com maior escolaridade, que trabalham fora de casa e tinham mamilos íntegros, a intervenção educativa influenciou para melhor autoeficácia na amamentação. O grupo de intervenção apresentou média mais elevada para autoeficácia na amamentação e maior frequência de aleitamento materno exclusivo. Conclusão: nas nutrizes internadas na maternidade estudada, a intervenção educativa influenciou para a manutenção da amamentação exclusiva nos $60^{\circ}$ primeiros dias após o parto.

Descritores: Amamentação. Educação em Saúde. Saúde Materno-infantil. Enfermagem.

Objective: to evaluate the nursing educational intervention for the promotion of the breastfeeding self-efficacy of nursing mothers hospitalized in a maternity hospital in northern Brazil. Method: quasi-experimental, longitudinal study, composed of an intervention group $(n=80)$ and observation group $(n=78)$. The educative intervention

\footnotetext{
Enfermeira e Obstetra. Mestre em Ciências da Saúde. Enfermeira da Prefeitura Municipal de Porto Velho. Porto Velho, Rondônia, Brasil. http://orcid.org/0000-00024255-1439.

Enfermeira. Doutora em Enfermagem em Saúde Pública. Professora Associada da Universidade Federal de Rondônia. Porto Velho, Rondônia, Brasil. http://orcid. org/0000-0002-1460-0803.

Enfermeira. Doutora em Ciências da Saúde. Professora Associada da Universidade Federal de Rondônia. Porto Velho, Rondônia, Brasil. http://orcid.org/0000-000 I $8900-6801$.

4 Estudante de Enfermagem. Universidade Federal de Rondônia. Porto Velho, Rondônia, Brasil. lerissaferreira@gmail.com. http://orcid.org/0000-0002-6949-098X

Estudante de Enfermagem. Universidade Federal de Rondônia. Porto Velho, Rondônia, Brasil.http://orcid.org/0000-0003-3057-1368.

Enfermeira. Doutora em Enfermagem. Professora Associada da Universidade Federal de Rondônia. Porto Velho, Rondônia, Brasil. http://orcid.org/0000-000 I-7238-5999.
} 
occurred through conversation wheel. Data collection occurred in the period from January to March 2018. Results: in the intervention group, nursing mothers showed high breastfeeding efficacy, whereas in the observation group, the efficacy was moderate. Among nursing mothers with more schooling, who work out of home and with intact nipples, the educative intervention influenced for better breastfeeding self-efficacy. The intervention group showed higher mean breastfeeding self-efficacy and increased frequency of breastfeeding. Conclusion: for the nursing mothers hospitalized in the maternity of study, the educative intervention influenced for the maintenance of exclusive breastfeeding within the 60 first days after delivery.

Descriptors: Breast Feeding. Health Education. Maternal and Child Health. Nursing.

Objetivo: evaluar la intervención educativa de enfermería para la promoción de la autoeficacia en amamantamiento en las madres lactantes en una maternidad del Norte de Brasil. Método: un estudio cuasi-experimental, longitudinal, compuesto por un grupo de intervención $(n=80)$ y un grupo observacional $(n=78)$. La intervención educativa ocurrió a través de rueda de conversación. La recogida de datos se produjo en el período de enero a marzo de 2018. Resultados: en el grupo de intervención, las madres lactantes mostraron una alta eficacia en amamantamiento, mientras que en el grupo observacional, la autoeficacia fue moderada. Entre las madres con mayor escolaridad, que trabajan fuera del hogar y con los pezones intactos, la intervención educativa influenció para una mejor autoeficacia en el amamantamiento. El grupo de intervención mostró mayor promedio para la autoeficacia en el amamantamiento y frecuencia aumentada del amamantamiento. Conclusión: para las madres lactantes en la maternidad estudiada, la intervención educativa influenció el mantenimiento del amamantamiento exclusivo en los 60 primeros días después del parto.

Descriptores: Lactancia Materna. Educación en Salud. Salud Materno-Infantil. Enfermeria.

\section{Introdução}

O aleitamento materno tem importância fundamental para o crescimento e o desenvolvimento adequados da criança, como também para a sua saúde física e psicológica. É de fato considerado em todo o mundo um dos pilares fundamentais para a promoção e proteção à saúde da criança, e seu impacto social pode ser quantificado por meio da redução de atendimentos médicos, hospitalizações e tratamentos medicamentosos, uma vez que a criança em amamentação tem menor risco de adoecer ${ }^{(1)}$.

O leite materno é o alimento mais adequado para a criança desde o nascimento até os primeiros anos de vida, contribuindo para a saúde das crianças e das mães ${ }^{(2)}$. Essa prática acarreta em benefícios ao desenvolvimento da criança desde o nascimento até a fase adulta ${ }^{(2)}$. A Organização Mundial da Saúde (OMS) recomenda o aleitamento materno exclusivo (AME) até aos seis meses e, posteriormente, a introdução da alimentação complementar mantendo o aleitamento materno (AM) até os dois anos ou mais ${ }^{(3)}$.

Um panorama mundial aponta que, no ano de 2015, o continente europeu tinha o menor índice global de amamentação e que apenas
13\% dos bebês até seis meses eram alimentados exclusivamente com leite materno. Em apenas 23 países encontrou-se pelo menos $60 \%$ de aleitamento materno exclusivo até seis meses, sendo que todos eram países em desenvolvimento ${ }^{(4)}$. Na Segunda Pesquisa de Prevalência de Aleitamento Materno Exclusivo realizado nas capitais brasileiras e no Distrito Federal, no ano de 2009, verificou-se prevalência de $41 \%$ de AME em menores de seis meses. Na Região Norte a média de AME foi de 66,2 dias e no município de Porto Velho, Rondônia, apenas 50,8\% das crianças estavam em aleitamento materno exclusivo com a idade de 60 dias $^{(5)}$.

As principais causas do desmame precoce estão relacionadas à introdução de outros alimentos na dieta do lactente antes do período recomendado; recusa do seio materno pela criança, que está diretamente relacionado com o posicionamento incorreto do recém-nascido no momento da amamentação; trabalho materno fora do domicílio; rejeição ao ato de amamentar pela própria mãe; doenças maternas e da criança; utilização de medicamentos pela mãe; impressão materna de que a criança não tem sua fome saciada com esse leite; e escassez de programas educativos eficientes ${ }^{(6)}$. 
Um dos aspectos que pode influenciar na manutenção da AME é a confiança materna na habilidade de amamentar. Esta habilidade se constrói mediante quatro fontes de informação que fundamentam a autoeficácia na amamentação: experiência pessoal; experiência vicária - observação de outras experiências; persuasão verbal - apoio e encorajamento de pessoas próximas; estado emocional e fisiológico - reações físicas e emocionais positivas no ato de amamentar $^{(7)}$. Diferentemente das variáveis não modificáveis ou pouco modificáveis que interferem na amamentação, como o estado marital, nível educacional e as condições socioeconômicas, a confiança materna é uma variável modificável. Conhecer o nível de confiança de uma puérpera permite identificar as mulheres de maior risco para o desmame precoce e realizar intervenções individualizadas quando for necessário ${ }^{(8)}$.

O suporte ao aleitamento materno é multidimensional, sendo necessário apoio prático, informativo e emocional $^{(9)}$. A educação em saúde é essencial para que as mulheres sintam-se preparadas para cuidar de seus filhos, incluindo a amamentação. Assim, o objeto desta pesquisa é compreender o impacto de ações de educação em saúde realizadas pela enfermagem na autoconfiança das mães em amamentar.

Sabe-se que o processo educacional, quando realizado periodicamente, pode contribuir para o autocuidado. Entretanto, ainda é pouco discutido se ações únicas, pontuais e no ambiente hospitalar podem trazer benefícios às puérperas. Portanto, entendemos que ainda há espaço no meio acadêmico para discutir a eficácia de diferentes ações educativas realizadas pelos profissionais da saúde na manutenção do AME.

Dessa forma, este estudo teve como objetivo avaliar a intervenção educativa de enfermagem para a promoção da autoeficácia em amamentação em nutrizes internadas no alojamento conjunto de uma maternidade do Norte do Brasil.

\section{Método}

Estudo do tipo quase experimental, longitudinal, realizado em uma maternidade municipal que atende mulheres para a assistência ao parto, ao pós-abortamento e ao planejamento familiar na cidade de Porto Velho, Rondônia, Brasil.

Para o cálculo amostral utilizou-se o Epi Infoß7.2.1.0, considerou-se a população de 4.084 puérperas admitidas na instituição no ano de 2017, nível de significância de 95\%, margem de erro de 5\%, e moderada ou alta autoeficácia de $87 \%$ dos sujeitos ${ }^{(10)}$. A amostra obtida foi de 167 participantes. Aplicando 10\% de perdas de dados, a amostra final desejada foi 184 nutrizes.

Foram incluídas puérperas com recém-nascidos vivos, internadas no alojamento conjunto, com no mínimo 6 horas e até 48 horas pós-parto, idade superior a 12 anos, residentes em Porto Velho, com telefone fixo ou celular e estar praticando AME durante a internação. Foram excluídos da pesquisa: as participantes que não estavam acordadas e lúcidas no momento da coleta de dados; as portadoras de vírus da imunodeficiência humana (HIV) ou hepatites; os recém-nascidos impossibilitados de serem amamentados devido a más-formações.

Os grupos de intervenção (ação educativa sobre amamentação exclusiva) e de observação (atendidos pela equipe multiprofissional da instituição conforme a rotina do serviço) foram compostos por meio de um sorteio das enfermarias. Dessa forma, todas as gestantes da enfermaria sorteada foram convidadas para comporem o grupo correspondente de intervenção ou de observação.

A intervenção e a coleta de dados foram realizadas por pesquisadores previamente treinados e ocorreu em momentos diferentes, não existindo comunicação entre as participantes dos dois grupos (intervenção ou observação).

O grupo de intervenção recebeu orientações sobre AME por meio de rodas de conversa, utilizando como apoio visual o álbum seriado "Promovendo o aleitamento materno"(11). Este álbum aborda os seguintes itens: a importância do aleitamento materno, livre demanda na amamentação, posições para amamentar, pega correta, tempo de mamada, ordenha e armazenamento e desvantagens do uso de bicos e chupetas. Após a aplicação do questionário e observados os problemas mamários, os pesquisadores 
auxiliaram no posicionamento do bebê, na pega correta para a mamada e ajudaram na realização de massagem mamária e ordenha, quando necessário. A intervenção educativa tinha duração de 20 a 30 minutos e foi apoiada na teoria de autoeficácia de Bandura ${ }^{(12)}$, que defende a ideia de que o indivíduo possui capacidades que lhes possibilitam utilizar símbolos (cognições) para realizar ação específica, necessária à obtenção de determinado resultado.

O período de coleta de dados no grupo observacional ocorreu durante oito dias consecutivos no mês de março de 2018. No grupo de intervenção foram 24 dias de coleta realizados diariamente nos meses de janeiro e fevereiro de 2018. A coleta nesse grupo foi realizada logo após a intervenção.

Para a coleta de dados utilizou-se um questionário com duas partes: informações sociodemográficas, história obstétrica e sobre a amamentação atual e anterior; e escala de avaliação da autoeficácia para amamentação Breastfeeding Self-Efficacy Scale - Short-Form (BSES-SF).

A escala reduzida BSES-SF foi validada no Brasil $^{(13)}$. A escala é constituída por 14 itens distribuídos em duas categorias de domínio: Domínio Relativo à Técnica - ações físicas que uma mãe deve realizar e que são necessárias para uma amamentação bem-sucedida; Domínio de Pensamentos Intrapessoais - percepções da mãe quanto à amamentação, incluindo atitudes e crenças relacionadas à amamentação bem-sucedida. Aos itens da escala são atribuídos escores que podem variar de 1 (discordo totalmente) a 5 (concordo totalmente) e perfazem um total de 14 a 70 pontos. Neste estudo a pesquisadora adaptou a pontuação para utilizar apenas dois escores da escala Likert para cada categoria: discordo (0 ponto), e concordo (1 ponto). Assim, a pontuação final das categorias foi: 0 a 5 pontos eficácia baixa, 6 a 10 pontos eficácia moderada, e 11 a 14 pontos eficácia alta na amamentação.

Este foi um estudo longitudinal, com seguimento das participantes por 60 dias após o parto, sendo realizado contato telefônico nos $7^{\circ}$, $15^{\circ}, 30^{\circ}, 45^{\circ}$, e $60^{\circ}$ dias. Justifica-se o ponto de corte do seguimento do estudo até a $8^{\mathrm{a}}$ semana pós-parto devido à constatação que a duração média da AME no Brasil é de 54,1 dias ${ }^{(5)}$.

Os dados foram processados no pacote estatístico Stata ${ }^{\circledR} 15.0$ e foi utilizado o teste t de Student considerando estatisticamente significante $\mathrm{p}<0,05$.

Este estudo foi previamente aprovado pelo Comitê de Ética em Pesquisa (CEP) do Núcleo de Saúde da Fundação Universidade Federal de Rondônia, seguindo os princípios da Resolução n. 466/12 do Conselho Nacional de Pesquisa ${ }^{(14)}$, em março de 2018, sob o Parecer n. 2.548.115. Os dados foram coletados após assinatura do Termo de Consentimento Livre e Esclarecido (TCLE) para maiores de 18 anos e o Termo de Assentimento Livre e Esclarecido (TALE) para as puérperas menores de 18 anos.

\section{Resultados}

Foram incluídas no estudo 184 nutrizes; desse total houve $13 \%(n=12)$ de perdas ou recusas no grupo de intervenção e 15\% (n=14) de perdas no grupo observacional e a amostra final foi de 158 participantes. A faixa etária predominante foi de 19 a 30 anos, porém 17\% das participantes tinham menos de 18 anos (Tabela 1). A maioria das puérperas tinha companheiro, não trabalhava fora de casa e tinha mais de nove anos de estudo. Quanto aos dados sobre a gestação, a maioria era multípara e teve parto do tipo vaginal.

Tabela 1 - Características sociodemográficas, obstétricas e sobre a amamentação. Porto Velho, Rondônia, Brasil -2017 (N=158)

(continua)

\begin{tabular}{l|c|c|c|c}
\hline \multirow{2}{*}{ Variáveis } & \multicolumn{2}{|c|}{ Grupo de Intervenção } & \multicolumn{2}{c}{ Grupo de Observação } \\
\cline { 2 - 5 } & $\mathbf{n = 8 0}$ & $\mathbf{\%}$ & $\mathbf{n = 7 8}$ & $\mathbf{\%}$ \\
\hline Faixa Etária & \multicolumn{3}{c}{} & \\
$<$ 18 anos & 17 & 21,2 & 10 & 12,8 \\
$19-24$ anos & 33 & 41,2 & 35 & 44,9 \\
$25-30$ anos & 22 & 27,5 & 24 & 30,8 \\
$31-35$ anos & 7 & 8,8 & 7 & 9,0 \\
$>36$ anos & 1 & 1,3 & 2 & 2,6
\end{tabular}


Tabela 1 - Características sociodemográficas, obstétricas e sobre a amamentação. Porto Velho, Rondônia, Brasil -2017 (N=158)

(conclusão)

\begin{tabular}{|c|c|c|c|c|}
\hline \multirow{2}{*}{ Variáveis } & \multicolumn{2}{|c|}{ Grupo de Intervenção } & \multicolumn{2}{|c|}{ Grupo de Observação } \\
\hline & $\mathbf{n}=\mathbf{8 0}$ & $\%$ & $n=78$ & $\%$ \\
\hline \multicolumn{5}{|l|}{ Estado Civil } \\
\hline Sem companheiro & 11 & 13,7 & 14 & 17,9 \\
\hline Com companheiro & 69 & 86,3 & 64 & 82,1 \\
\hline \multicolumn{5}{|l|}{ Trabalho fora de casa } \\
\hline Não & 58 & 72,5 & 62 & 80,8 \\
\hline Sim & 22 & 27,5 & 16 & 19,2 \\
\hline \multicolumn{5}{|l|}{ Escolaridade } \\
\hline$\leq 9$ anos & 25 & 31,3 & 20 & 25,6 \\
\hline$>9$ anos & 55 & 68,8 & 58 & 74,4 \\
\hline \multicolumn{5}{|l|}{ Número de Gestações } \\
\hline Primigesta & 29 & 36,3 & 31 & 39,7 \\
\hline Multigesta & 51 & 66,8 & 47 & 60,3 \\
\hline \multicolumn{5}{|l|}{ Tipo de Parto } \\
\hline Cesárea & 21 & 26,3 & 22 & 28,2 \\
\hline Vaginal & 59 & 73,7 & 56 & 71,8 \\
\hline \multicolumn{5}{|l|}{ Tipos de Mamilos } \\
\hline Plano/Semiplano & 15 & 18,8 & 8 & 10,3 \\
\hline Protuso/Semiprotuso & 65 & 81,2 & 69 & 88,4 \\
\hline Invertido/Pseudo Invertido & - & - & 1 & 1,3 \\
\hline \multicolumn{5}{|l|}{ Condições das mamas } \\
\hline Flácida & 72 & 90,0 & 57 & 73,1 \\
\hline Globosa & 5 & 6,25 & 18 & 23,1 \\
\hline Ingurgitada & 3 & 3,75 & 3 & 3,8 \\
\hline \multicolumn{5}{|l|}{ Condições dos Mamilos } \\
\hline Íntegros & 62 & 77,5 & 43 & 55,1 \\
\hline Lesionados & 18 & 22,5 & 35 & 44,9 \\
\hline \multicolumn{5}{|l|}{$\begin{array}{l}\text { Problema de amamentação } \\
\text { anterior }(n=98)\end{array}$} \\
\hline Não & 37 & 72,5 & 32 & 68,0 \\
\hline $\operatorname{Sim}$ & 14 & 27,5 & 15 & 32,0 \\
\hline \multicolumn{5}{|c|}{ Problemas na amamentação atual } \\
\hline Não & 57 & 71,2 & 40 & 51,3 \\
\hline $\operatorname{Sim}$ & 23 & 28,8 & 38 & 48,7 \\
\hline \multicolumn{5}{|l|}{ Tipo de Problema } \\
\hline Pega Incorreta & 21 & 26,3 & 32 & 41,0 \\
\hline Pouco Leite & 2 & 2,5 & 6 & 7,7 \\
\hline Sem problema & 57 & 71,2 & 40 & 51,3 \\
\hline \multicolumn{5}{|c|}{ Orientações sobre amamentação } \\
\hline Não & - & - & 23 & 29,5 \\
\hline $\operatorname{Sim}$ & 80 & 100 & 55 & 70,5 \\
\hline \multicolumn{5}{|l|}{ Local da Orientação } \\
\hline Pré-natal & - & - & 8 & 10,3 \\
\hline Maternidade & 42 & 52,5 & 29 & 37,2 \\
\hline ré-natal e maternidade & 38 & 47,5 & 18 & 23,1 \\
\hline Não recebeu orientação & - & - & 23 & 29,5 \\
\hline
\end{tabular}

Fonte: Elaboração própria.

Nota: Sinal convencional utilizado:

- Dado numérico igual a zero não resultante de arredondamento. 
Observou-se no momento da entrevista que apenas uma puérpera do grupo de intervenção não estava com presença de leite/colostro. A maioria das puérperas tinha mamas flácidas e mamilos do tipo protusos ou semiprotusos. Os mamilos estavam lesionados em 44,9\% das puérperas no grupo de observação e em 22,5\% das mulheres no grupo de intervenção.

Quanto à experiência anterior de amamentar, 72,5\% do grupo de intervenção e 68,0\% do grupo de observação informaram que não tiveram problemas para amamentar o filho anterior. Com relação à amamentação do filho atual, $28,8 \%$ do grupo de intervenção e 48,7\% do grupo de observação referiram problemas para amamentar o bebê, sendo que o tipo de problema predominante foi "pega incorreta".

Sobre orientações em aleitamento materno, foi realizada uma intervenção educativa em tipo "roda de conversa" com as puérperas, e, desse grupo, $47,5 \%$ já haviam recebido orientações durante o pré-natal. No grupo de observação, $29,5 \%$ informaram não ter recebido nenhuma orientação sobre amamentação e 37,2\% informaram que receberam orientações sobre a amamentação apenas na maternidade.

Todas as nutrizes do grupo de intervenção apresentaram eficácia alta na amamentação (Tabela 2). Entre as participantes do grupo de observação, aquelas que possuíam o tipo de mamilo plano/protuso (Média=10,14; DV=2,52) e invertidos (Média 10), mamas do tipo ingurgitadas (Média=9,8; $\mathrm{DP}=3,76$ ), problemas na amamentação atual (Média=10,59; DP=2,69) e nutrizes com problemas de "pega incorreta" (Média=10,44; DP=2,7) tiveram eficácia moderada na amamentação. Neste estudo não foram encontradas participantes que indicaram baixa eficácia.

Apenas entre os extremos de idade e mulheres com escolaridade inferior a nove anos de estudos, a média de autoeficácia entre o grupo de intervenção não foi superior ao do grupo de observação. Entretanto, as diferenças entre esses grupos não foram estatisticamente significativas.

Tabela 2 - Média de autoeficácia em amamentar e características sociodemográficas, obstétricas e sobre amamentação. Porto Velho, Rondônia, Brasil - 2017 (N=158) (continua)

\begin{tabular}{|c|c|c|c|c|c|}
\hline \multirow[t]{2}{*}{ Variáveis } & \multicolumn{2}{|c|}{$\begin{array}{c}\text { Grupo de Intervenção } \\
(n=80)\end{array}$} & \multicolumn{2}{|c|}{$\begin{array}{l}\text { Grupo de Observação } \\
(n=78)\end{array}$} & \multirow[t]{2}{*}{ Valor de $p$} \\
\hline & Média & (DP) & Média & (DP) & \\
\hline \multicolumn{6}{|l|}{ Faixa Etária } \\
\hline$<18$ anos & 12,23 & 1,78 & 12,28 & 2,70 & 0,47 \\
\hline 19-24 anos & 12,14 & 1,60 & 11,31 & 2,7 & 0,08 \\
\hline 25-30 anos & 11,94 & 1,51 & 11,52 & 2,13 & 0,23 \\
\hline $31-35$ anos & 12,16 & 0,74 & 11,50 & 2,08 & 0,28 \\
\hline$>36$ anos & 12 & - & 12,6 & 1,5 & - \\
\hline \multicolumn{6}{|l|}{ Estado Civil } \\
\hline Sem companheiro & 12,20 & 2,30 & 11,42 & 2,35 & 0,22 \\
\hline Com companheiro & 12,11 & 1,52 & 11,54 & 2,35 & 0,05 \\
\hline \multicolumn{6}{|l|}{ Trabalho fora de casa } \\
\hline Não & 11,98 & 1,64 & 11,66 & 2,19 & 0,18 \\
\hline $\operatorname{Sim}$ & 12,47 & 1,6 & 11,00 & 2,8 & 0,04 \\
\hline \multicolumn{6}{|l|}{ Escolaridade } \\
\hline$\leq 9$ anos & 11,83 & 1,76 & 12,22 & 1,55 & 0,22 \\
\hline$>9$ anos & 12,25 & 1,57 & 11,34 & 2,5 & 0,01 \\
\hline \multicolumn{6}{|l|}{ Número de Gestações } \\
\hline Primigesta & 12,25 & 1,75 & 11,34 & 2,37 & 0,05 \\
\hline Multigesta & 12,04 & 1,57 & 11,64 & 2,32 & 0,17 \\
\hline \multicolumn{6}{|l|}{ Tipo de Parto } \\
\hline Cesárea & 12,28 & 1,90 & 11,31 & 2,83 & 0,09 \\
\hline Normal & 12,05 & 1,53 & 11,61 & 2,14 & 0,11 \\
\hline
\end{tabular}


Tabela 2 - Média de autoeficácia em amamentar e características sociodemográficas, obstétricas e sobre amamentação. Porto Velho, Rondônia, Brasil - 2017 (N=158)

(conclusão)

\begin{tabular}{|c|c|c|c|c|c|}
\hline \multirow[t]{2}{*}{ Variáveis } & \multicolumn{2}{|c|}{$\begin{array}{c}\text { Grupo de Intervenção } \\
(\mathbf{n = 8 0 )}\end{array}$} & \multicolumn{2}{|c|}{$\begin{array}{c}\text { Grupo de Observação } \\
(n=78)\end{array}$} & \multirow[t]{2}{*}{ Valor de $\mathbf{p}$} \\
\hline & Média & (DP) & Média & (DP) & \\
\hline \multicolumn{6}{|l|}{ Tipos de Mamilos } \\
\hline Plano/Semiplano & 12,00 & 1,64 & 10,14 & 2,52 & 0,05 \\
\hline Protuso/Semiprotuso & 12,20 & 1,69 & 11,69 & 2,29 & 0,08 \\
\hline Invertido & - & - & 10 & - & - \\
\hline \multicolumn{6}{|l|}{ Condições dos Mamilos } \\
\hline Íntegros & 12,10 & 1,64 & 11,39 & 2,34 & 0,04 \\
\hline Lesionados & 12,16 & 1,65 & 11,69 & 2,35 & 0,20 \\
\hline \multicolumn{6}{|l|}{ Condições das mamas } \\
\hline Flácidas & 12,11 & 1,60 & 11,48 & 2,45 & 0,08 \\
\hline Globosas & 12,80 & 1,30 & 11,84 & 1,84 & 0,09 \\
\hline Ingurgitadas & 11,00 & 2,60 & 9,80 & 3,76 & 0,30 \\
\hline \multicolumn{6}{|c|}{ Problema de amamentação anterior } \\
\hline Não & 12,02 & 1,61 & 11,45 & 2,51 & 0,13 \\
\hline $\operatorname{Sim}$ & 12,08 & 1,50 & 12,07 & 1,85 & 0,49 \\
\hline \multicolumn{6}{|c|}{ Problemas na amamentação atual } \\
\hline Não & 12,33 & 1,52 & 12,23 & 1,73 & 0,38 \\
\hline Sim & 11,59 & 1,78 & 10,59 & 2,69 & 0,05 \\
\hline \multicolumn{6}{|l|}{ Tipo de Problema } \\
\hline Pega Incorreta & 11,40 & 1,75 & 10,44 & 2,7 & 0,07 \\
\hline Pouco Leite & 13,50 & 0,70 & 12,66 & 1,96 & 0,20 \\
\hline Sem problema & 12,33 & 1,52 & 12,15 & 1,75 & 0,29 \\
\hline \multicolumn{6}{|c|}{ Orientações sobre amamentação } \\
\hline Não & - & - & 11,63 & 1,86 & - \\
\hline Sim & 12,12 & 1,63 & 11,48 & 2,52 & 0,05 \\
\hline \multicolumn{6}{|l|}{ Local da Orientação } \\
\hline Pré-natal & - & - & 11,42 & 1,61 & - \\
\hline Maternidade & 12,21 & 1,54 & 11,35 & 2,90 & 0,08 \\
\hline Pré-Natal e maternidade & 12,02 & 1,73 & 11,75 & 2,22 & 0,30 \\
\hline Não recebeu & - & - & 11,63 & 1,86 & - \\
\hline
\end{tabular}

Fonte: Elaboração própria.

Nota: Sinal convencional utilizado:

- Dado numérico igual a zero não resultante de arredondamento.

Entre as mulheres com escolaridade superior a nove anos (Média 12,25 versus 11,34; $\mathrm{p}=0,01$ ) e que trabalham fora de casa (Média 12,47 versus $11,0 ; p=0,04)$ encontrou-se diferenças significativas entre a média de autoeficácia. Também foi encontrada melhor autoeficácia entre mulheres com mamilos íntegros no grupo de intervenção (Média 12,10 versus 11,39; $\mathrm{p}=0,04$ ).

A intervenção educativa foi importante na manutenção da amamentação exclusiva ao longo do tempo. Em todo o período de seguimento (60 dias), o grupo de intervenção teve maior frequência de amamentação exclusiva quando comparada ao grupo de observação (Tabela 3). A média de autoeficácia relatada durante a internação hospitalar foi superior entre o grupo de intervenção e manteve-se estatisticamente significativa nos cinco momentos do seguimento. 


\begin{tabular}{|c|c|c|c|c|c|}
\hline \multirow[t]{2}{*}{ Variáveis } & \multicolumn{2}{|c|}{ Grupo de Intervenção } & \multicolumn{2}{|c|}{ Grupo de Observação } & \multirow{2}{*}{$\begin{array}{l}\text { Valor } \\
\text { de } p\end{array}$} \\
\hline & n (\%) & Média (DP) & n (\%) & Média (DP) & \\
\hline \multicolumn{6}{|c|}{ Amamentação exclusiva $7^{\circ}$ dias } \\
\hline Sim & $79(98,7)$ & $12,13(1,64)$ & $75(96,1)$ & $11,52(2,34)$ & 0,03 \\
\hline Não & $1(0,3)$ & $11,00(\ldots)$ & $3(3,9)$ & $11,66(2,51)$ & $\ldots$ \\
\hline \multicolumn{6}{|c|}{ Amamentação exclusiva $15^{\circ}$ dias } \\
\hline $\operatorname{Sim}$ & $74(91,2)$ & $12,14(1,64)$ & $71(91,0)$ & $11,40(2,36)$ & 0,01 \\
\hline Não & $6(7,5)$ & $11,85(1,67)$ & $7(9,0)$ & $12,71(1,79)$ & 0,18 \\
\hline \multicolumn{6}{|c|}{ Amamentação exclusiva $30^{\circ}$ dias } \\
\hline $\operatorname{Sim}$ & $74(92,5)$ & $12,14(1,65)$ & $63(80,7)$ & $11,45(2,32)$ & 0,03 \\
\hline Não & $6(7,5)$ & $11,8(1,48)$ & $15(19,3)$ & $11,8(2,45)$ & 0,50 \\
\hline \multicolumn{6}{|c|}{ Amamentação exclusiva $45^{\circ}$ dias } \\
\hline Sim & $70(87,5)$ & $12,14(1,66)$ & $42(53,9)$ & $10,74(2,53)$ & $<0,01$ \\
\hline Não & $10(12,5)$ & $12,00(1,49)$ & $36(46,1)$ & $12,36(1,79)$ & 0,26 \\
\hline \multicolumn{6}{|c|}{ Amamentação exclusiva $60^{\circ}$ dias } \\
\hline Sim & $67(83,75)$ & $12,11(1,67)$ & $36(46,15)$ & $10,66(2,59)$ & $<0,01$ \\
\hline Não & $13(16,25)$ & $12,15(1,46)$ & $42(53,85)$ & $12,21(1,86)$ & 0,44 \\
\hline
\end{tabular}

Fonte: Elaboração própria.

Nota: Sinal convencional utilizado:

... dado numérico não disponível.

\section{Discussão}

No grupo de intervenção as médias indicaram que as nutrizes apresentaram maior nível de autoeficácia para a amamentação quando comparado ao grupo de observação. A intervenção educativa contribuiu para elevar o nível de autoeficácia entre as participantes com escolaridade superior a nove anos de estudo, que trabalham fora de casa e tinham mamilos íntegros.

Neste estudo, 17\% das puérperas tinham menos de 18 anos, corroborando a tendência nacional, na qual, no ano de 2012, a frequência de gestação na adolescência foi $21,1 \%{ }^{(15)}$. O parto entre adolescentes foi a primeira causa de internação hospitalar de mulheres na faixa etária entre 10 e 19 anos durante o ano de 2012 no Brasil $^{(15)}$. A gravidez na adolescência é a principal causa de morte de mulheres jovens entre 15 e 19 anos nos países em desenvolvimento e está associada à baixa escolaridade, ao abandono escolar, ao baixo nível socioeconômico e ao pré-natal inadequado ${ }^{(16)}$. Além disso, a idade inferior a vinte anos é fator importante para o desmame precoce - a mãe adolescente mostra uma frequência maior de desmame quando comparado com mães adultas ${ }^{(17)}$. Encontrou-se maior média na autoeficácia para amamentação no grupo de intervenção, com exceção entre as puérperas em limites de idade, isto é, adolescentes ou mulheres com mais de 35 anos. Estudo realizado em 2007 afirma que quanto maior a idade das mulheres, melhor desempenho na amamentação ${ }^{(13)}$.

A maioria das participantes tinha companheiro. Sabe-se que a presença do parceiro durante a fase de aleitamento materno contribui para sua manutenção ${ }^{(11)}$. Estudo realizado no Canadá observou que mulheres solteiras tinham maior risco para interrupção precoce do aleitamento materno quando comparado às casadas ${ }^{(18)}$. Outro estudo, conduzido em Uganda, África Ocidental, com 84 puérperas que frequentavam um hospital de ensino, apontou que 6 de cada 10 mulheres apresentaram elevada autoeficácia para a amamentação, e os fatores associados foram a presença de companheiro e receber apoio dos profissionais de saúde à amamentação durante a internação para o parto $^{(19)}$.

Neste estudo, encontrou-se a predominância de multíparas e a maioria delas indicou que não houve problemas na amamentação de seus filhos anteriores. A experiência prévia na amamentação interfere na segurança para amamentar o seu próximo bebê e essa interferência pode ser positiva ou negativa ${ }^{(20)}$.

Quanto ao tipo de parto, a maioria das mulheres teve parto vaginal, com um quantitativo de cesáreas abaixo de 30\%. Essa baixa frequência 
de cesáreas deve-se principalmente porque a maternidade possuía o título de Hospital Amigo da Criança, que preconiza que o parto vaginal seja a via preferencial para os nascimentos, e este fato contribui para o início precoce da amamentação ${ }^{(21-22)}$.

Neste estudo, puérperas com mamilos íntegros tiveram média de autoeficácia mais elevada no grupo de intervenção quando comparado ao grupo de observação, indicando que essas puérperas estiveram mais propensas a aprenderem sobre amamentação e, consequentemente, sentiram-se seguras para a manutenção da AME. Mamas ingurgitadas, mamilos planos e invertidos, lesionados ou doloridos, preocupação materna com a qualidade e quantidade de leite, estresse e ansiedade são fatores que interferem negativamente no aleitamento materno ${ }^{(23)}$. A presença de dor e ansiedade materna dificulta a amamentação e cabe à equipe multiprofissional oferecer auxílio. A equipe de saúde deve observar os sinais e sintomas que as puérperas possam apresentar durante a internação, reforçando sobre o processo de produção do leite e apojadura e referenciá-las para grupos de aleitamento materno, evitando assim a interrupção da amamentação ${ }^{(23)}$.

Algumas mulheres não receberam previamente qualquer informação sobre a amamentação durante o pré-natal ou mesmo na maternidade. Sabe-se que orientações adequadas durante o pré-natal favorecem a autoconfiança em amamentar ${ }^{(20)}$. O acompanhamento por um profissional de saúde beneficia a preparação da mãe e dos familiares para o aleitamento materno. As gestantes devem ser informadas sobre os benefícios da prática, as desvantagens de outros leites e as técnicas de amamentação para aumentar as habilidades e autoconfiança materna ${ }^{(20)}$.

A intervenção educativa realizada em apenas um momento durante a internação na maternidade influenciou na manutenção da amamentação exclusiva durante os dois meses subsequentes. O grupo de intervenção apresentou média mais elevada para autoeficácia declarada durante a internação hospitalar, e esse fato refletiu na manutenção do aleitamento materno exclusivo até 60 dias.
Uma coorte prospectiva realizada no sul do Brasil, no ano de 2012, demostrou que hospitais com título de Amigo da Criança, que estimulavam a amamentação precoce e exclusiva durante a internação na maternidade, tiveram maior média de tempo de AME durante os quatro primeiros meses de vida do recém-nascido quando comparado a outros hospitais ${ }^{(21)}$. Um estudo quase experimental realizado em Taiwan, com 214 participantes, encontrou que a realização de um único encontro sobre amamentação no formato de roda de conversa foi capaz de melhorar a autoeficácia das puérperas e a AME durante as primeiras quatro a cinco semanas de vida do recém-nascido quando comparado àquelas que receberam o atendimento rotineiro de saúde ${ }^{(24)}$. Outra pesquisa, realizada em nove maternidades da Dinamarca, com 1476 participantes, avaliou o impacto de uma ação educativa usando um panfleto e aconselhamento individualizado às puérperas durante a internação ${ }^{(25)}$. Os autores encontraram que a intervenção não afetou a autoeficácia da amamentação, no entanto, menos crianças foram readmitidas uma semana pós-natal em comparação com o grupo de referência, e em seis meses após o nascimento, mais crianças foram amamentadas exclusivamente e com maior frequência no grupo intervenção ${ }^{(25)}$.

Entre as potencialidades deste estudo podemos destacar o uso da escala de avaliação da autoeficácia para amamentação Breastfeeding Self-Efficacy Scale - Short-Form (BSES-SF). É uma escala reconhecida e usada mundialmente, tornando possível a comparação entre os resultados dos estudos. Além disso, estudo realizado na França com 149 participantes enfatiza que essa escala pode ser usada para identificar precocemente, seja na maternidade ou no pré-natal, as mães que não se sentem capazes de amamentar ${ }^{(26)}$.

\section{Conclusão}

A escala de autoeficácia em amamentação apresentou-se como um instrumento fácil e rápido para identificar o perfil das nutrizes, colaborando com o planejamento individualizado 
de ações educativas, visando melhorar a confiança da nutriz e a manutenção da AME. A intervenção educativa, mesmo que realizada em um único momento e de modo breve, mas focada nas nutrizes, baseada na teoria da autoeficácia e usando o método de roda de conversa, foi o suficiente para elevar a autoeficácia das participantes em amamentar e contribuiu para a manutenção da AME nos dois primeiros meses de vida da criança.

Outras formas de intervenção podem ser experimentadas visando à manutenção da AME durante os primeiros seis meses de vida da criança como, por exemplo, o uso das tecnologias da informação para envio de mensagens de textos pelo celular ou uso de aplicativos para aprendizagem autodirigida sobre amamentação. Constituem meios de apoio rápidos, baratos e de amplo acesso às puérperas, mas que ainda precisam ser testados quanto a sua efetividade.

\section{Colaborações:}

1 - concepção, projeto, análise e interpretação dos dados: Sandra Maria Schultz, Kátia Fernanda Alves Moreira e Priscilla Perez da Silva Pereira;

2 - redação do artigo e revisão crítica relevante do conteúdo intelectual: Sandra Maria Schultz, Kátia Fernanda Alves Moreira, Priscilla Perez da Silva Pereira, Lerissa Nauana Ferreira, Marcos Antônio Sales Rodrigues e Daiana Evangelista Rodrigues Fernandes;

3 - aprovação final da versão a ser publicada: Sandra Maria Schultz, Kátia Fernanda Alves Moreira, Priscilla Perez da Silva Pereira, Lerissa Nauana Ferreira, Marcos Antônio Sales Rodrigues e Daiana Evangelista Rodrigues Fernandes.

\section{Referências}

1. Nunes LM. Importância do aleitamento materno na atualidade. Bol Cient Pediatr [Internet]. 2015 [cited 2020 Feb 3];4(3):55-8. Available from: https://www.sprs.com.br/sprs2013/bancoimg/ 160529234034bcped_v4_n3_a2.pdf

2. Lopes WC, Marques FKS, Oliveira CF, Rodrigues JA, Silveira MF, Caldeira AP, et al. Alimentação de crianças nos primeiros dois anos de vida. Rev paul pediatr. 2018;36(2):164-70. DOI:https://doi.org/10.1590/1984-0462/;2018;36;2;00004

3. World Health Organization. Guideline: protecting, promoting and supporting breastfeeding in facilities providing maternity and newborn services [Internet]. Geneva (CH); 2017 [cited 2020 Jan 4]. Available from: http://www.who.int/nutrition/ publications/guidelines/breastfeeding-facilitiesmaternity-newborn/en/

4. Organização Mundial da Saúde. Departamento de Saúde Reprodutiva e Pesquisa. Declaração da OMS sobre Taxas de Cesáreas [Internet]. Genebra (CH); 2015 [cited 2017 Oct 2]. Available from: http:// apps.who.int/iris/bitstream/10665/161442/3/ WHO_RHR_15.02_por.pdf

5. Brasil. Ministério da Saúde. Secretaria de Atenção à Saúde. Departamento de Ações Programáticas e Estratégicas. II Pesquisa de Prevalência de Aleitamento Materno nas Capitais Brasileiras e Distrito Federal [Internet]. Brasília (DF); 2009 [cited 2019 Oct 20]. Available from: http:// bvsms.saude.gov.br/bvs/publicacoes/pesquisa_ prevalencia_aleitamento_materno.pdf

6. Alvarenga SC, Castro SD, Leite FMC, Brandão MAG, Zandonade E, Primo CC. Fatores que influenciam no desmame precoce. Aquichan. 2017;17(1):93-103. DOI: 10.5294/aqui.2017.17.1.9

7. Oriá MOB, Ximenes LB. Tradução e adaptação cultural da Breastfeeding Self-Efficacy Scale para O português. Acta paul enferm. 2010;23(2):230-8. DOI: https://doi.org/10.1590/S0103-21002010000200013

8. Oriá MO, Ximenes LB, Almeida PC, Glick DF, Dennis CL. Psychometric assessment of the Brazilian version of the Breastfeeding Self-Efficacy Scale. Public Health Nurse. 2009;26(6):574-83. DOI: $10.1111 /$ j.1525-1446.2009.00817.x

9. Ferrari AG, Cherer EQ, Piccinini CA. Aspectos subjetivos da amamentação e desmame: evidência em três casos. Psic.: Teor. e Pesq. 2017;33:e33411. DOI: https://doi.org/10.1590/0102.3772e33411

10. Guimarães CMS, Conde RG, Gomes-Sponholz FAG, Oriá MOB, Monteiro JCS. Fatores relacionados à autoeficácia na amamentação no pós-parto imediato entre puérperas adolescentes. Acta paul enferm. 2017;30(1):109-15. DOI: http://dx.doi. org/10.1590/1982-0194201700016

11. Brasil. Ministério da Saúde. Secretaria de Atenção à Saúde. Promovendo o aleitamento materno [Internet]. 2a ed. Brasília (DF); 2007 [cited 2016 Nov 1]. Available from: http: //www.unicef.org/ brazil/pt/aleitamento.pdf 
12. Bandura A. Self-efficacy: toward a unifying theory of behavioral change. Psychol Rev. 1977;84(2):191-215. DOI: https://doi.org/10.1016/0146-6402(78)90002$4 \mathrm{Get}$ rights and content

13. Javorski M, Rodrigues AJ, Dodt RCM, Almeida PC, Leal LP, Ximenes LB. Effects of an educational technology on self-efficacy for breastfeeding and practice of exclusive breastfeeding. Rev Esc Enferm USP. 2018;52:e3329. DOI: 10.1590/ S1980-220X2017031803329

14. Brasil. Ministério da Saúde. Conselho Nacional de Saúde. Resolução n. 466, de 12 de dezembro de 2012. Aprova diretrizes e normas regulamentadoras de pesquisa envolvendo seres humanos [Internet]. Brasília (DF); 2012 [cited 2016 Oct 22]. Available from: http://bvsms.saude.gov.br/bvs/saudelegis/ cns/2013/res0466_12_12_2012.html

15. Fiedler MW, Araújo A, Souza MCC. A prevenção da gravidez na adolescência na visão de adolescentes. Texto contexto enferm. 2015;24(1):30-7. DOI: https://doi.org/10.1590/010407072015000130014

16. Brasil. Ministério da Saúde. Centro Brasileiro de Análise e Planejamento. Pesquisa nacional de demografia e saúde da criança e da mulher PNDS 2006: dimensões do processo reprodutivo e da saúde da criança [Internet]. Brasília (DF); 2009 [cited 2017 Mar 24]. Available from: http://bvsms. saude.gov.br/bvs/publicacoes/pnds_crianca_ mulher.pdf

17. Maranhão TA, Gomes KRO, Nunes LB, Moura LNB. Fatores associados ao aleitamento materno exclusivo entre mães adolescentes. Cad saúde colet. 2015;23(2):132-9. DOI: 10.1590/1414-462X201500020072

18. Brown LF, Thoyre S, Pridham K, Schubert C. The Mother-Infant Feeding Tool. J Obstet, Gynecol Neonatal Nurs. 2014;38(4):491-503. DOI: $10.1111 / \mathrm{j} .1552-6909.2009 .01047 . \mathrm{x}$

19. Nankumbi J, Mukama AA, Ngabirano TD. Predictors of breastfeeding self-efficacy among women attending an urban postnatal clinic, Uganda. Nurs Open. 2019;6(3):765-71. DOI: 10.1002/nop2.257

20. Rodrigues AP, Padoin SMM, Guido LA, Lopes LFD. Fatores do pré-natal e do puerpério que interferem na autoeficácia em amamentação. Esc Anna Nery. 2014;18(2):257-61. DOI: $10.5935 / 1414-8145.20140037$

21. Brasil. Ministério da Saúde. Iniciativa Hospital Amigo da Criança (HAC) [Internet]. Brasília (DF); 2017 [cited 2020 May 20]. Available from: https:// www.saude.gov.br/artigos/41186-iniciativahospital-amigo-da-crianca-ihac

22. Lamounier JA, Chaves RG, Rego MAS, Bouzada MCF. Iniciativa Hospital Amigo da Criança: 25 Anos de Experiência no Brasil. Rev paul pediatr. 2019;37(4):486-93. DOI: 10.1590/1984-0462/;2019;37;4;00004

23. Uchoa JL, Rodrigues AP, Joventino ES, Almeida PC, Oriá MOB, Ximenes LB. Autoeficácia em amamentar de mulheres no pré-natal e no pós-parto: estudo longitudinal. Rev enferm UFSM. 2016;6(1):10-20. DOI: $10.5902 / 2179769217687$

24. Lee YH, Chang GL, Chang HY. Effects of education and support groups organized by IBCLCs in early postpartum on breastfeeding. Midwifery. 2019;75:5-11. DOI: 10.1016/j.midw.2019.03.023

25. Nilsson IMS, Strandberg-Larsen K, Knight CH, Hansen AV, Kronborg H. Focused breastfeeding counselling improves short- and long-term success in an early-discharge setting: A cluster-randomized study. Matern Child Nutr. 2017 Oct;13(4):e12432. DOI: $10.1111 / \mathrm{mcn} .12432$

26. Dégrange M, Delebarre M, Turck D, Mestdagh B, Storme L, Deruelle P, et al. Is self-confidence a factor for successful breastfeeding? Arch Pediatr. 2015 Jul;22(7):708-17. DOI: 10.1016/ j.arcped.2015.04.008

Recebido: 25 de março de 2020 Aprovado: 7 de maio de 2020 Publicado: 1 de junho de 2020

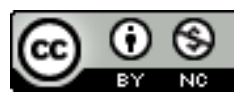

A Revista Baiana de Enfermagem utiliza a Licença Creative Commons - Atribuição-NãoComercial 4.0 Internacional. https://creativecommons.org/licenses/by-nc/4.0/

Este artigo é de acesso aberto distribuído sob os termos da Licença Creative Commons (CC BY-NC). Esta licença permite que outros remixem, adaptem e criem a partir do seu trabalho para fins não comerciais. Embora os novos trabalhos tenham de lhe atribuir o devido crédito e não possam ser usados para fins comerciais, os usuários não têm de licenciar esses trabalhos derivados sob os mesmos termos. 